\title{
Correction to: How urbanization affects multiple dimensions of biodiversity in tropical butterfly assemblages
}

\author{
Cristiano Agra Iserhard ${ }^{1} \cdot$ Leandro Duarte ${ }^{2}$. Noemy Seraphim ${ }^{3,4}$. \\ André Victor Lucci Freitas ${ }^{4}$
}

Published online: 21 January 2021

(c) Springer Nature B.V. 2021

\section{Correction to: Biodiversity and Conservation (2019) 28:621-638 https://doi.org/10.1007/s10531-018-1678-8}

In the first paragraph of page 627, under section 'Diversity measures and functional composition', the sentence "Positive or negative SES.MPD/MFD values indicate phylogenetic/ functional clustering or overdispersion of species in the fragment, respectively (Webb et al. 2002)." was published incorrectly. The correct sentence should read "Positive or negative SES.MPD/MFD values indicate phylogenetic/functional overdispersion or clustering of species in the fragment, respectively (Webb et al. 2002)."

Publisher's Note Springer Nature remains neutral with regard to jurisdictional claims in published maps and institutional affiliations.

The original article can be found online at https://doi.org/10.1007/s10531-018-1678-8.

Cristiano Agra Iserhard

cristianoagra@yahoo.com.br

1 Programa de Pós-Graduação em Biologia Animal, Departamento de Ecologia, Zoologia eGenética, Instituto de Biologia, Universidade Federal de Pelotas, Mail Box 354, Pelotas, Rio Grande do Sul 96160-000, Brazil

2 Departamento de Ecologia, Universidade Federal do Rio Grande do Sul, Porto Alegre, Rio Grande do Sul, Brazil

3 Instituto Federal de Educação, Ciência e Tecnologia de São Paulo, campus, Campinas, São Paulo, Brazil

4 Departamento de Biologia Animal and Museu de Zoologia, Universidade Estadual de Campinas, Campinas, São Paulo, Brazil 